\title{
On a General Iterative Method for the Approximate Solution of Linear Operator Equations
}

\author{
By W. V. Petryshyn*
}

1. Introduction. A number of iterative procedures have been developed for the approximate solution of a linear operator equation of the form $A u=f$, where $f$ is a given element in some suitably normed linear space and $A$ is either a matrix, an integral, or an abstract operator in this space.

The purpose of this paper is to unify and extend investigations of finite algebraic systems by von Mises and Polaczek [10], Cesari and Picone [4], Quade [12], Keller [8,9] and others [19]; integral equations of the Fredholm type by Neumann [5], Wiarda [18], Bückner [2, 3], Wagner [17], Samuelson [14], and Fridman [7]; and operator equations in abstract Hilbert or Banach spaces by Schönberg [15], Rall [13], Bialy [1], and Petryshyn [11].

This generalization and unification of various methods in terms of conditions for convergence and error estimates is accomplished by studying a rather general iteration procedure of which the above methods are special cases. It is hoped that the procedure presented here can be used as a basis for possible discovery of new iterative methods when applied to concrete problems.

2. Existence, convergence, and error estimate. Let $X$ denote a complete real or complex normed linear vector space, $P$ a linear bounded operator in $X$, and $R(P)$ the range space of $P$. We say that $P$ is invertible if $P$ has a bounded inverse $P^{-1}$ on $R(P)$, i.e., $P^{-1}$ is such that $P^{-1} P u=u$ for all $u \in X$ and $P P^{-1} v=v$ for all $v \in R(P)$. If, in addition, $R(P)=X$, then $P$ is called continuously invertible. Let $m$ and $M$ be non-negative real numbers defined by

$$
m(P)=\underset{u \neq 0}{\operatorname{g.l} . b .} \frac{\|P u\|}{\|u\|}, \quad M(P)=\underset{u \neq 0}{\text { l.u.b. }} \frac{\|P u\|}{\|u\|}
$$

for all $u \in X$. It is known [ $\|$ ] that $P$ is invertible if and only if $m(P)>0$. Furthermore, if $m(P)>0$, then $\left\|P^{-1}\right\|=\frac{1}{m}$ and $m(P) M\left(P^{-1}\right)=1$.

Our problem is to develop a general iteration procedure for the approximate solution of the operator equation

$$
A u=f,
$$

where $f$ is a given element in $X$ and $A$ is a given linear bounded operator in $X$. Our first step in this direction is to investigate the existence and the uniqueness

Received April 27, 1962. The work presented in this paper is partly supported by the AEC Computing and Applied Mathematics Center, Courant Institute of Mathematical Sciences, New York University, Atomic Energy Commission and the National Science Foundation.

* Temporary member at the Courant Institute of Mathematical Sciences. 
of the solution of equation (1) when $A$ satisfies certain conditions stated in Theorem 1 or Corollary 1 below.

THEOREM 1. Equation (1) has a unique solution $u^{*}$ for every $f$ in $X$ if and only if there exists a continuously invertible operator $C$ and $a$ bounded linear operator $B$ with an inverse $B^{-1}$ having the property that the series

$$
\sum_{i=0}^{\infty} T^{i} g
$$

converges for every $g$ in $X$, where $T=C^{-1} L$ and $L=C-B A$. The solution $u^{*}$ in this case is given by the sum of (2).

Proof. Let $f$ be an arbitrary element in $X$ and $C$ and $B$ be some operators having the above property. If we put $g=C^{-1} B f$, then in view of the completeness of $X$ and our hypothesis the series (2) converges to some element $u^{*}$ in $X$. Moreover, $u^{*}$ is a solution of equation (1) for if $u^{*}=\sum_{i=0}^{\infty} T^{i} g$, then $T u^{*}=u^{*}-g$ or equivalently $C^{-1} B A u^{*}=C^{-1} B f$. This and our conditions on $B$ and $C$ imply that $A u^{*}=f$; i.e., $u^{*}$ is a solution of (1). To show that $u^{*}$ is a unique solution of equation (1) let us suppose that equation (1) has another solution $u \neq u^{*}$. Then $v=u-u^{*} \neq 0$ and $A v=0$ or $B A v=0$. This implies that $C v-B A v=L v=C v$ or equivalently that $(1-T) v=0$ from which it follows that the operator $(I-T)$ has no inverse. This, however, is a contradiction to the hypothesis (2) for, if the series (2) converges for every $g$ in $X$, then $(I-T)^{-1}$ exists and

$$
(I-T)^{-1} g=\sum_{i=0}^{\infty} T^{i} g
$$

for every $g$ in $X$. In fact, if $(I-T) h=0$ for some $h \neq 0$, then $h=T h=T^{2} h=$ $\cdots=T^{n} h$. Put $h_{n+1}=\sum_{i=0}^{n} T^{i} h$. By hypothesis (2), $\lim _{n} h_{n+1}=\sum_{i=0}^{\infty} T^{i} h$ exists. Now $\lim _{n}\left(\frac{1}{n+1} h_{n+1}\right)=\lim _{n} \frac{1}{n+1} \cdot \lim h_{n+1}=0 \cdot \lim _{n} h_{n+1}=0$. But $\lim _{n}\left(\frac{1}{n+1} h_{n+1}\right)=\lim _{n} h=h$. Thus $h=0$. This implies that $(I-T)^{-1}$ exists and that $u=u^{*}$ is a unique solution of equation (1). Since $T$ is bounded and $u^{*}=\sum_{i=0}^{\infty} T^{i} g,(1-T) u^{*}=\lim _{n} \sum_{i=0}^{n} T^{i} g-\lim _{n} \sum_{i=1}^{n} T^{i} g=\lim _{n} g=g$ whence we conclude that $u^{*}=(1-T)^{-1} g=\sum_{i=0}^{\infty} T^{i} g$. This proves the first part of Theorem 1.

To prove the converse of Theorem 1 note that if equation (1) has a unique solution $u^{*}$ for every $f$ in $X$, then $A$ is continuously invertible. We may thus choose, for example, $C$ to be an arbitrary continuously invertible operator in $X$ and $B=$ $\eta C A^{-1}$, where $\eta$ is a complex number such that $|1-\eta|<1$. Then $B^{-1}=\frac{1}{\eta} A C^{-1}$ exists and $T=C^{-1} L=C^{-1}(C-B A)=1-\eta$. Since $\|T\|=|1-\eta|<1$ and $\left\|T^{n}\right\| \leqq\|T\|^{n}$ the series $(2), \sum_{i=0}^{\infty} T^{i} g=\sum_{i=0}^{\infty}(1-\eta)^{i} g$, converges to some element in $X$ for any $g$ in $X$. Suppose we denote by $u$ the sum of the latter series when $g=C^{-1} B f$. Since, on the other hand, $\sum_{i=0}^{\infty}(1-\eta)^{i} g=\frac{1}{\eta} g$, we have $u=\frac{1}{\eta} g=\frac{1}{\eta} C^{-1} B f=\frac{1}{\eta} C^{-1} \eta C A^{-1} f=A^{-1} f$. This shows that $u=u^{*}$ and completes the proof of the theorem. 
Let us observe that in some practical cases it may turn out that the verification of the very general condition (2) is not easy. In these cases the following corollary can be used in which condition (2) is replaced by conditions that are simpler but more restrictive and in some cases easier to verify.

CoROllary 1. The assertion of Theorem 1 remains valid if condition (2) is replaced by one of the following three conditions

$$
\begin{aligned}
\varlimsup_{n} \sqrt[n]{\left\|T^{n} g\right\|} & <1, \text { for every } g \in X, \\
\lim _{n} \sqrt[n]{\left\|T^{n}\right\|} & <1, \\
\|T\| & <1,
\end{aligned}
$$

whose degree of restriction increases in the given order.

Proof. We see from the proof of Theorem 1 that it is sufficient to show that $(2.3) \Rightarrow(2.2) \Rightarrow(2.1) \Rightarrow(2)$. Let $\sigma(T)$ denote the spectrum of $T$, i.e., the set of all complex numbers $\lambda$ for which the operator $(\lambda I-T)$ is not continuously invertible, and $r(T)=\sup _{\lambda_{\sigma \in \sigma}(T)}|\lambda|$, the spectral radius of $T$. It is known [16] that

$$
r(T)=\lim _{n} \sqrt[n]{\left\|T^{n}\right\|}
$$

and that for every positive integer $n$

$$
r(T) \leqq \sqrt[n]{\left\|T^{n}\right\|}
$$

Thus, in view of (4) with $n=1,(2.3) \Rightarrow(2.2)$. To show that $(2.2) \Rightarrow(2.1)$, we consider the series

$$
\sum_{i=0}^{\infty} \mu^{i} T^{i} g
$$

where $\mu$ is a complex number and $g$ is any element in $X$. The radius of convergence $r$ of (5) is given by the formula

$$
r=\frac{1}{\overline{\lim _{n}} \sqrt[n]{\left\|T^{n} g\right\|}}
$$

with the property that (5) converges for $|\mu|<r$ and diverges for $|\mu|>r$. Since for each $n$ and $g$ in $X,\left\|T^{n} g\right\| \leqq\left\|T^{n}\right\|\|g\|$ we easily derive from (3) and (6) that

$$
r(T) \geqq \frac{1}{r}\left(=\varlimsup_{n} \sqrt[n]{\left\|T^{n} g\right\|}\right) .
$$

Thus, by (7) and (6), if $r(T)<1$, then $\overline{\lim }_{n} \sqrt[n]{\left\|T^{n} g\right\|}<1$ for each $g$; i.e., $(2.2) \Rightarrow$ (2.1). Finally, if $\varlimsup_{n} \sqrt[n]{\left\|T^{n} g\right\|}<1$ for every $g$ in $X$, then $r>1$ and thus by the above mentioned property of $r$ the series $\sum_{i=0}^{\infty} T^{i} g$, which is (5) with $\mu=1$, converges in $X$.

Remark 1. In case $C=I$ and $B$ is continuously invertible, Theorem 1, under the more restrictive condition (2.3), was proved by Rall [13]. 
Theorem 2. If for every $f$ in $X$ equation (1) possesses a unique solution $u^{*}$ in $X$, then the sequence $\left\{u_{n+1}\right\}$ of iterants determined by the process

$$
C u_{n+1}=L u_{n}+f_{1} \text {, }
$$$$
n=0,1,2, \cdots,
$$

where $C$ and $L$ are the same as in Theorem 1 and $f_{1}=B f$, converges to the solution $u^{*}$ of equation (1) for any initial approximation $u_{0}$ in $H$ if and only if condition (2) of Theorem 1 is satisfied. In case of convergence the error estimate is given by

$$
\left\|u_{n+1}-u^{*}\right\| \leqq\left\|(B A)^{-1} L\right\|\left\|u_{n+1}-u_{n}\right\|
$$

or by a less precise but a more practical estimate

$$
\left\|u_{n+1}-u^{*}\right\| \leqq \frac{M(L)}{m(B A)}\left\|u_{n+1}-u_{n}\right\|
$$

Proof. Let the sequence $\left\{u_{n+1}\right\}$ be determined by (8) or equivalently by

$$
u_{n+1}=T u_{n}+g,
$$

where $g=C^{-1} f_{1}$ and $T=C^{-1} L$. We find from (11) by induction that

$$
u_{n+1}=\sum_{i=0}^{n} T^{i} g+T^{n+1} u_{0}
$$

Equation (12) implies that the sequence $\left\{u_{n+1}\right\}$ converges if and only if the series (2), $\sum_{i=0}^{\infty} T^{i} g$, converges. By Theorem 1 , the limit $u^{*}=\lim _{n} u_{n+1}$, which is the same as the sum of the series (2) with $g=C^{-1} f_{1}$, satisfies equation (1) and is independent of the choice of $u_{0}$ for condition (2) implies that $\lim _{n} T^{n+1} u_{0}=0$ for any $u_{0} \in X$, i.e., $(2.1) \Rightarrow(2)$.

To derive estimates (7) and (10) let $u^{*}$ be the exact solution and $u_{n+1}$ an approximate solution of (1) determined by (8). Subtracting $L u_{n+1}$ from both sides of (8) we get $C u_{n+1}-L u_{n+1}=L\left(u_{n}-u_{n+1}\right)+f_{1}$ or $B A u_{n+1}=L\left(u_{n}-u_{n+1}\right)+f_{1}$. On the other hand, $B A u^{*}=f_{1}$ and, therefore, subtracting the corresponding sides in the last two equations we obtain

$$
B A\left(u_{n+1}-u^{*}\right)=L\left(u_{n}-u_{n+1}\right) .
$$

Note that $\left(2^{\prime}\right)$ implies that $m(B A)>0$ for, by $\left(2^{\prime}\right)$, the operator $(I-T)^{-1}=$ $\left[I-\left(I-C^{-1} B A\right)\right]^{-1}=\left(C^{-1} B A\right)^{-1}$ exists on all of $H$ and is bounded. From this we see that $(B A)$ has a bounded inverse given by $\left(C^{-1} B A\right)^{-1} C^{-1}$. The error estimates (9) and (10) follow now immediately from (13) and the properties of $m$ and $M$ as defined in $(a)$. This completes the proof of Theorem 2.

Corollary 2. For convergence of the sequence $\left\{u_{n+1}\right\}$ determined by (8) to the solution $u^{*}$ of (1) for a given $f$ in $X$ it is sufficient that there exist operators $C$ and $B$ satisfying any one of the more restrictive conditions (2.1), (2.2), or (2.3) of Corollary 1. The error estimates in this case are the same as in Theorem 2.

At the end of this section let us remark that in case $C$ is invertible, $B$ possesses an inverse $B^{-1}, R(C) \supseteq R(B A)$, and $X_{0}$ denotes the subspace of $X$ consisting of all elements $g$ in $X$ of the form $g=C^{-1} B h, h \in R(A)$, then from the arguments of the first part of Theorem 1 and Theorem 2 we derive

Corollary 3. If, for a given $f$ in $X$, equation (1) is solvable, then for any initial approximation $u_{0}$ in $X_{0}$ the sequence $\left\{u_{n+1}\right\}$ determined by $(8)$ converges to the solution 
of (1) if the series $\sum_{i=0}^{\infty} T^{i} g$ converges for every $g$ in $X_{0}$, where $T=C^{-1} L$ with $C$ and $B$ having the properties specified in the last paragraph.

3. Special Cases. The general method (8) is not precise until the choice of $C$ and $B$ has been made. In this section we classify a number of suggested iterative methods for the solution of (1) by specifying operators $C$ and $B$ in (8) or (11). At the same time, some of the methods that were investigated and used before only for finite matrix equations will thus be extended to operator equations in infinite dimensional normed linear spaces. In order not to repeat ourselves each time when we specify a particular method it will be assumed, in what follows, that equation (1) is solvable and that in various special cases the resulting operator $T=C^{-1}(C-B A)=I-C^{-1} B A$ satisfies at least one of the conditions of Section 1 , thus ensuring the convergence of $\left\{u_{n+1}\right\}$. Furthermore, if for a given special method of (8) or (11) the operator $T$ satisfies the corresponding condition of Theorem 2 or any one of Corollary 2, then for each special method considered below the corresponding error estimates (9) and (10) remain valid.

Method 1a. If $C=I$ and $B=\alpha>0$, where $I$ is the identity operator in $X$ and $\alpha$ is a real parameter, then (8) reduces to the standard iteration

$$
u_{n+1}=(I-\alpha A) u_{n}+\alpha f
$$

which converges to the solution of (1) if $\alpha$ is so chosen that $T=I-\alpha A$ satisfies the conditions of Section 1.

In particular, if we assume that $\sigma(A)$ contains only eigenvalues $\lambda$ of $A$, then $\sigma(T)$ also contains only eigenvalues $\mu$ of the form $\mu=1-\alpha \lambda$ and, therefore, exactly as in the author's paper [11] one shows that convergent schemes (8.1a) can be found if $R_{e} \lambda>0$. Indeed, in that case a suitable $\alpha$ can be determined corresponding to any circle in the $(x, y)$-plane $(\lambda=x+i y)$ which passes through the point $(0,0)$, has a center on the real $x$-axis, and which is such that all eigenvalues $\lambda$ of $A$ lie in its interior. If $\left(\frac{1}{\alpha}, 0\right)$ is the center of such a circle; i.e., of $\left(x-\frac{1}{\alpha}\right)^{2}+y^{2}=$ $\frac{1}{\alpha^{2}}$, then this value of $\alpha$ gives a convergent scheme. If, in addition, $A$ is symmetric and $\lambda>0$, then from the above remark (also see [11]) one easily derives that (8.1a) converges for any $\alpha$ in the interval

$$
0<\alpha<\frac{2}{\|A\|},
$$

where the number $\lambda=\|A\|$ is the largest eigenvalue of $A$ [16].

Let us note that in case $A$ is an integral operator determined by a square summable symmetric and positive definite kernel in $L_{2}(a, b)$ the convergence of (8.1a) with $\alpha$ satisfying (14) was proved by Fridman [7]. Bialy [1] extended his results to the case when $A$ is a non-negative symmetric operator in a Hilbert space $H$ by showing that for $\alpha$ satisfying (14) scheme (8.1a) converges to a solution of (1) if and only if (1) is solvable.

Remark 2. The above discussion applies fully to the case when $R_{e} \lambda<0$ if $\lambda$ are complex and to the case when $\lambda<0$ if $\lambda$ are real and $A$ is symmetric.

Method $1 b$. If $A$ is not symmetric, then instead of (8.1a) one may use

$$
u_{n+1}=\left(I-\alpha A^{*} A\right) u_{n}+\alpha A^{*} f
$$


which is (11) with $C=\alpha^{-1}, \quad B=A^{*}$, and $T=I-\alpha A^{*} A$. Formula (8.1b) was proposed for matrix equations by Quade [12] who proved its convergence for any $\alpha$ in

$$
0<\alpha<\frac{2}{\left\|A^{*} A\right\|}
$$

Bialy extended the applicability of $(8.1 \mathrm{~b})$ to operator equations in $H$. Note that since $A^{*} A$ is a non-negative operator in $H$ and $\left\|A^{*} A\right\|=\|A\|^{2}$ the procedure (8.1b) is (8.1a) applied to the equation $A^{*} A u=A^{*} f$ which, as can be easily proved, is equivalent to equation (1) since (1) is solvable.

Method 1c. If $A$ is a symmetric but not positive integral operator of the form $A=I-\lambda K$, then Bückner [3] suggested the procedure

$$
u_{n+1}=\left(I-(-1)^{n-1} \beta A\right) u_{n}+(-1)^{n-1} \beta f
$$

which, as was also shown by Bialy for an arbitrary symmetric operator $A$ in $H$, converges for all $\beta$ in the interval

$$
0<\beta<\frac{\sqrt{2}}{\|A\|} .
$$

It follows from (8.1c) that its equivalent iteration formula is

$$
u_{n+1}=\left(I-\beta^{2} A A\right) u_{n-1}+\beta^{2} A f
$$

which is (11) with $C=\beta^{-2}, \quad B=A$, and $T=I-\beta^{2} A A$ for the subsequences $\left\{u_{2 n}\right\}$ and $\left\{u_{2 n+1}\right\}$. The inequality (16) implies (15) with $\alpha=\beta^{2}$. Since equation (1) is solvable, both subsequences converge to the same solution of (1).

Method 2. If $A$ is a non-singular matrix, then Cesari and Picone [4] proposed a method of the form

$$
C u_{n+1}=(C-A) u_{n}+f
$$

where $C$ is an arbitrary non-singular matrix. This is the process (8) if we take $B=I$ and $C$ an arbitrary continuously invertible operator in space $X$. Theorem 2 and Corollary 2 extend the applicability of (8.2) to linear operator equations in $X$ and supply it with the corresponding error estimates provided that

$$
T=C^{-1}(C-A)
$$

satisfies the specified condition. Recently Frey [6] generalized the process (8.2) to a special kind of nonlinear operator equations in Banach space under the assumption that $u_{0}=C^{-1} f$ and $\|T\|<1$, where $T$ is in this case nonlinear.

Method 3. If $C=I$ and $B$ is an arbitrary continuously invertible operator in $X$, then (8) reduces to the method

$$
u_{n+1}=(I-B A) u_{n}+B f
$$

whose convergence and error estimate were obtained by Rall [13] under the assumption that $T=I-B A$ satisfies the stringent condition (2.3). In the case in which $A$ is a nonsingular matrix and $B=D^{-1}$, where $D$ is a diagonal matrix, method (8.3) was first used by von Mises and Polaczek-Geringer [10]. Our Theorem 2 and Corollary 2 extend the validity of method (8.3) to operator equations for 
which the operator $T$ satisfies the weaker conditions (2.2), (2.1), or even the general condition (2).

Method 4. Let $A$ be of the form $A=D-S-Q$ and $\omega$ be a positive real number. If we take $C=D-\omega S$ and $B=\omega I$, then $L=(1-\omega) D+\omega Q$ and (8) reduces to the generalized overrelaxation iterative method

$$
(D-\omega S) u_{n+1}=[(1-\omega) D+\omega Q] u_{n}+\omega f
$$

whose convergence and error estimates (9) and (10) were obtained by the author [11] in the case in which $X=H$ under the assumption that $A$ is $K$-positive definite; i.e., there exists a linear operator $K$ and a constant $\beta>0$ such that $(A u, K u) \geqq$ $\beta\|K u\|^{2}$ for all $u \in H,(D u, K u)$ is real for all $u, G(\omega)=\frac{2-\omega}{\omega} D-S+Q^{*}$ (where $Q^{*}$ is the adjoint of $Q$ ) is $K$-positive definite, and $D-\omega S$ is continuously invertible. $\dagger$ Under these conditions it is shown that

$$
T=(D-\omega S)^{-1}[(1-\omega) D+\omega Q]
$$

satisfies condition (2.2) †† provided that $K$ and $\sigma(T)$ satisfy the conditions specified in [11]. In the case in which $Q=S^{*}, K=I$, and $A$ is a finite matrix, (8.4) was thoroughly investigated by Young [19].

Method 5. If $A=D-S-S^{*}$ and if we choose $C=D-S$ and $B=I$, then $L=S^{*}$ and (8) becomes the Gauss-Seidel method

$$
(D-S) u_{n}=S^{*} u_{n}+f
$$

for which Section 1 supplies the convergence and error estimates when (8.5) is applied to operator equations in $X$. For example, if $D=I$, Fredholm integral equations of the second kind in the $L_{p}(a, b)$-spaces $1 \leqq p<\infty$ can be split in this way. This can also be done for the space $C(a, b)$.

Method 6. Let $A=N-P$, where $N$ is continuously invertible, and $\alpha \neq-1$ be a real number. If we choose $C=(1+\alpha) N$ and $B=I$, then $L=P+\alpha N$ and (8) reduces to the procedure

$$
(I+\alpha) N u_{n+1}=(P+\alpha N) u_{n}+f
$$

proposed by Keller [9] for finite matrix equations and extended by the author [11] to operator equations in $H$ for the case when $\sigma\left(N^{-1} P\right)$ contains only eigenvalues $\lambda$ of finite multiplicity such that either $\lambda<1$ or $\lambda>1$ if $\lambda$ are real and either $R_{e} \lambda<1$ or $R_{\theta} \lambda>1$ if $\lambda$ are complex.

Method 7. Suppose $A=I-W-V$ and $\alpha$ and $\beta$ are two real non-zero parameters. In case $W V=V W$ and $W$ and $V$ are finite symmetric matrices Keller [8] studied the following method

$$
(\alpha I-\beta W) u_{n+1}=[(\alpha-1) I-(\beta-1) W+V] u_{n}+f .
$$

$\dagger$ The cases when $A$ is only $K$-positive and $K$-non-negative as well as the problem of optimum parameter $\omega$ for (8.4), are also considered in [11].

$\dagger \dagger$ Added in proof: The results containing much more general necessary and sufficient conditions for the spectrum $\sigma(T)$ of $T(\omega)$ to lie in the interior of the unit circle were since obtained by the author and will be contained in an article to appear in the Proceedings of Amer. Math. Soc. 
In view of Theorem 2 and Corollary 2, the method (8.7) is also applicable to operator equations in $X$ since it is (8) with $C=\alpha I-\beta W, B=I$, and

$$
L=(\alpha-1) I-(\beta-1) W+V=C-A
$$

provided, of course, that $C$ and $T=C^{-1} L=I-(\alpha-\beta W)^{-1} A$ satisfy the corresponding conditions for suitably chosen $\alpha$ and $\beta$.

This will be the case if, for example, we assume that $X$ is a Hilbert space $H$; the operators $W$ and $V$ are completely continuous, symmetric, and commutative; the number $\frac{\alpha}{\beta}$ is not an eigenvalue of $W ; A$ is positive definite; and the set of eigenvectors of $W$ and of $V$ is complete in $H$. Indeed, if $W$ and $V$ satisfy these properties, then $C-\beta\left(\frac{\alpha}{\beta} I-W\right)$ is continuously invertible and furthermore one can generalize to the Hilbert space $H$ the following lemma which allows us to extend the arguments and the results $t$ Keller [8] to the case when (8.7) is applied to the solution of the operator equation (1) in $H$ in which $A=I-W-V$.

Lemma. If $V$ and $W$ in $H$ sasisfy the above conditions, then they possess a complete set of common eigenvectors $\left\{\phi_{i}\right\}_{1}{ }^{\infty}$ such that $W \phi_{i}=\xi_{i} \phi_{i}$ and $V \phi_{i}=\eta_{i} \phi_{i}, i=1,2, \cdots$, where $\xi_{i}$ and $\eta_{i}$ are the corresponding eigenvaleus of $W$ and $V$.

Proof. Since $W$ is symmetric and completely continuous each of its eigenvalues $\xi_{i}$ is real and has a finite multipilcity $n_{i}$. Suppose that $\left\{\xi_{i}\right\}$, where each $\xi_{i}$ appears $n_{i}$ times, are so ordered that $\left\{\left|\xi_{i}\right|\right\}$ is a non-increasing sequence. Let $H_{i}$ be the eigenspaces of $W$ corresponding to the eigenvalues $\xi_{i}$; i.e., $H_{i}$ is the $n_{i}$-dimensional set of all $u$ in $H$ such that $W u=\xi_{i} u$, which is the same as to say that $H_{i}$ is the null-space of the operator $W_{i}=W-\xi_{i} I$. It is known [16] that $H_{i}$ is orthogonal to $H_{j}$ for $i \neq j$ and that the direct sum of $H_{i}$ is the whole space $H$.

We shall now construct a complete set of eigenvectors $\left\{\phi_{i}\right\}_{1}^{\infty}$ common to both $W$ and $V$. Let us fix $i$, say $i=1$. It is obvious that $W V=V W$ implies that $W_{i} V=$ $V W_{i}$. Hence, the null-space $H_{1}$ of $W_{1}$ and the range $R_{1}$ of $W_{1}$ are invariant under $V$; i.e., $V H_{1} \subset H_{1}$ and $V R_{1} \subset R_{1}$. Since $R_{1}$ is an orthogonal complement of $H_{1}$ in $H, H=H_{1} \oplus R_{1}$, the pair $\left(H_{1}, R_{1}\right)$ reduces $V$ and, therefore, $V$ can be represented on $H_{1}$ as an $n_{1} \times n_{1}$ symmetric matrix. This implies that $V$ considered in $H_{1}$ possesses $n_{1}$ eigenvectors $\phi_{1}, \phi_{2}, \cdots, \phi_{n_{1}}$ which span $H_{1}$ and can be chosen to be orthonormal. The elements $\phi_{1}, \phi_{2}, \cdots, \phi_{n_{1}}$ are also eigenvectors of $W$ since by assumption all vectors of $H_{1}$ are eigenvectors of $W$ corresponding to the eigenvalue $\xi_{1}$. Now, considered only on $R_{1}=H \ominus H_{1}$, the operators $W$ and $V$ have the same property as originally on $H$. We may therefore proceed with this process by applying it to $R_{1}$ instead of $H$ and replacing $H_{1}$ by $H_{2}$ and thus obtain a common set of eigenvectors $\phi_{n_{1}+1}, \phi_{n_{1}+2}, \cdots, \phi_{n_{2}}$ of $W$ and $V$ in $H_{2}$. Continuing this process sequentially for $i=1,2,3, \cdots$, we obtain a complete sequence of eigenvalues $\phi_{1}, \cdots, \phi_{n_{1}}, \phi_{n_{1}+1}, \cdots, \phi_{n_{2}}, \cdots$, common to both $W$ and $V$.

In view of our lemma one can extend the procedure of Keller to show that for any positive definite operator $A=I-W-V$ there exist open regions in the $(x, y)$-plane, $\dagger$ where $x \equiv \frac{\alpha}{\beta}$ and $y \equiv \frac{1}{\alpha}$, such that for all $(x, y)$ in these regions the operator $C=\alpha I-\beta W$ is continuously invertible and $T=I-(\alpha I-\beta W)^{-1} A$ satisfies condition (2.2).

$\dagger$ For the description of these regions and other details see [8]. 
For the rest of this section we shall assume that $A=I-\eta K$, where $\eta$ is a numerical parameter and $K$ acts in $X$. In what follows we shall, for the sake of completeness, reformulate in terms of our conditions the iterative methods surveyed by Rall [13].

Method 8. The simplest procedure is that of Neuman [5]

$$
u_{n+1}=\eta K u_{n}+f
$$

which is (8) with $C=I, B=I$, and $T=\eta K$. The two practically useful conditions on $T$ are (2.2), which in our case reduces to the requirement that $|\eta|<\begin{gathered}1 \\ r(K)\end{gathered}$ and the more restrictive condition $(2.3):|\eta|<\frac{1}{\|K\|}$. In case $K$ is completely continuous and symmetric these conditions reduce to the same requirement

$$
|\eta|<\left|\eta_{1}\right| \text {, }
$$

where $\eta_{1}$ is, in absolute sense, the smallest characteristic value of $K$.

Method 9 . If $K$ is a positive definite and symmetric integral operator condition (17) was removed by Wiarda [18] who used the iteration

$$
u_{n+1}=[\theta I+(1-\theta) \eta K] u_{n}+(1-\theta) f, \quad \theta \neq 1 .
$$

Formula $(8.9)$ is (8) with $C=I, B=(1-\theta) I$, and $T=\theta I+(1-\theta) \eta K$. Bückner [2] extended the investigations of Wiarda to nonsymmetric integral equations while Schönberg [15] generalized their results to the operator equations in a Banach space with $T$ satisfying condition (2.1).

Method 10. If $B=I$ and $C^{-1}=\frac{1}{x-\eta K x}$, where $K$ is an integral operator in a suitably chosen space $X$, and $x=1$, then (11) becomes the method proposed by Wagner [17]

$$
u_{n+1}=\left(I-C^{-1} A\right) u_{n}+C^{-1} f .
$$

Method 11. As the final special case we consider the method

$$
u_{n+1}=[I-(I+J) A] u_{n}+(1+J) f
$$

studied by Samuelson [14]. Formula (8.11) is (8) if we choose $C=I, B=I+J$, where $J$ is such an operator that $T=I-(I+J) A$ satisfies any one of our conditions in Section 1. It was suggested by Samuelson that $\dot{J}$ should be taken to be an approximation to the resolvent $G_{\eta}$ of $\eta K$. Let us note that since

$$
T=\left[A^{-1}-(I+J)\right] A=\left(G_{\eta}-\dot{J}\right) A
$$

it follows from the properties of spectral radii [16] that if $K \dot{J}=\dot{J} K$, then

$$
T=\left(G_{\eta}-J\right) A=A\left(G_{\eta}-J\right) \quad \text { and } \quad r(T) \leqq r\left(G_{\eta}-J\right) \cdot r(A) .
$$

This shows that $T$ satisfies condition (2.2) if $J$ is so chosen that $r\left(G_{\eta}-J\right)<\frac{1}{r(A)}$.

New York University

New York, New York

1. H. BiALy, "Iterative Behandlung linearer Funktionalgleichungen," Archive Rational Mech. Anal., 4(2), 1959, p. 166-176. 
2. H. BückNer, "A special method of successive approximations for Fredholm integral equations," Duke Math. J., 15, 1948, p. 197-206.

3. H. BüCKNER, "Ein unbeschränkt anwendbarer Iterationsverfahren für Fredholmsche Integralgleichungen," Math. Nachr., Berlin 2, 1949, p. 304-313.

4. L. Cessari, "Sulla risoluzione dei sistemi di equazioni lineari per approssimazioni successive," Atti Accad. Naz. Lincei, Rend. Cl. Sci. Fiz. Mat. Natur. VI. S. 25, 1937, p. 422-428.

5. R. Courant \& D. Hilbert, "Methoden der mathematischen Physik," 2 ed. v. 1, Berlin, Springer, 1931.

6. T. FREY, "Úber ein neues Iterationsverfahren zur Lösung von Integral- und Integrodifferentialgleichungen," PICC, Symposium, Rome, 1960, p. 384-387.

7. V. M. Fridman, "A method of successive approximation for Fredholm integral equations of the first kind," Uspehi Mat. Nauk, 11, 1956, p. 233-234.

8. H. B. KELLER, "Special block iterations with applications to Laplace and biharmonic difference equations," SIAM Rev. 2, 1960, p. 277-287.

9. H. B. Keller, Numerical methods. Lecture Notes, New York Univ., 1961.

10. R. von Mises \& U. H. Pollaczex-Geiringer. "Practische Vorfahren der Gleichungsauflösung," $Z$. Angew. Math. Mech., 9, 1929, p. 58-77 and 152-164.

11. W. V. Petryshyn, "The generalized overrelaxation method for the approximate solution of linear operator equations in Hilbert space," (to be published in SIAM J.).

12. W. QUADE, "Auflösung linearer Gleichungen durch Matrizeniterationen," Ber. Math.Tagung, Tübingen, 1946, p. 123-124.

13. L. B. RALL, "Error bounds for iterative solutions of Fredholm integral equations," Pacific J. Math., 5, 1955, p. 977-986.

14. P. A. SAmúlson, "Rapidly converging solutions to integral equations," J. Math. Phys., 31, 1953, p. 276-286.

15. M. Schö́nBerg, "Sur la methode d'iteration de Wiarda et de Bückner pour la resolution de Fredholm," Acad. Roy. Belgique, Bull. Cl. Sci., 37, 1951, p. 1941-1156 and 1952, p. 38 and p. 154-167.

16. A. E. TAYLOR, Introduction to functional analysis. Wiley, New York, 1957.

17. C. WAGNER, "On the solution of Fredholm integral equation of the second kind by iteration," J. Math. Phys., 30, 1951, p. 23-30.

18. G. WIARDA, Integralgleichungen unter besonderer Berücksichtigung der Anwendungen, Leipzig, 1930.

19. D. Young, "Iterative methods for solving partial difference equations of elliptic type," Trans. Amer. Math. Soc., 76, 1954, p. 92-111. 